\title{
Transport: Referees 2019
}

The following is a list of referees who have reviewed papers for Transport between 1 December 2018 and 30 November 2019.

The Institution of Civil Engineers is very grateful for their assistance.

We are continually looking for suitable reviewers for papers submitted to Transport. Papers published in the Proceedings of the ICE must be submitted to at least two independent referees to judge accuracy, style, impact, importance and interest.

If you are interested in reviewing articles on any topic related to transport, please submit your name, qualifications or CV, and

\begin{tabular}{|c|c|}
\hline Vahid Abolhasannejad & Mengqiu Cao \\
\hline Anwaar Ahmed & Halim Ceylan \\
\hline Huseyin Akbulut & Sai Chand \\
\hline Mir Ali & Guozhu Cheng \\
\hline Mazen Al-Kheetan & Muhammed Yasin Codur \\
\hline Borja Alonso & Jennifer Cooke \\
\hline Oruc Altintasi & Seosamh Costello \\
\hline Jiri Ambros & Brian Counter \\
\hline Sai Anjana & Li Cui \\
\hline Alex Apeagyei & Andre Dantas \\
\hline Justice Appiah & Pawan Deep \\
\hline Stephen Arhin & Gareth Dennis \\
\hline John Armstrong & Tomas Echaveguren \\
\hline Vittorio Astarita & Paul Edwards \\
\hline Ahmet Atalay & Morteza Esmaeili \\
\hline Erel Avineri & William Frith \\
\hline Kay Axhausen & Matthew Frost \\
\hline Metin Aydın & Ilgin Gökaşar \\
\hline Hazi Azamathulla & Fabio Galatioto \\
\hline Richard Bailey & Uneb Gazder \\
\hline Osman Bayrak & Bidisha Ghosh \\
\hline Justin Bishop & James Grenfell \\
\hline Thomas Bishop & Marco Guerrieri \\
\hline Simon Bowers & Ismail Bulent Gundogdu \\
\hline Annabel Bradbury & Ankit Gupta \\
\hline Peter Braithwaite & Martin Higginson \\
\hline Michael Burrow & John Hine \\
\hline John Cairns & John Hodgson \\
\hline Francisco Calvo & Bing Huang \\
\hline Ben Campbell & Timothy Hutchinson \\
\hline
\end{tabular}

areas of expertise. We are in need of individuals who will agree to review papers in a timely fashion (within 3 to 4 weeks of receipt) and provide confidential feedback to the Editorial Advisory Panel concerning the quality of the paper and any suggested revisions that would be appropriate.

If you are such a person, please contact Kirsten Buchanan (tel.: +44 (0)207 665 2204; e-mail: kirsten.buchanan@ice.org.uk) for more information on the referee process.

Morteza Jalili Qazizadeh

Guoqing Jing

Francois Jullemier

Sakdirat Kaewunruen

Sivakumar Kandasami

Xenia Karekla

Christos Katrakazas

Misbah Khan

Artur Kierzkowski

Murali Krishnan

S. Vasantha Kumar

Dina Kuttah

Jan-Mou Li

Tao Ma

Luca Mantecchini

Antonio Marcon

Arash Mazaheri

Emmerentian Mbabazi

Kevin McGhee

Michael McNerney

Rui Micaelo

Miomir Miljković

Mario Mongiardini

Galen Murton

Pooya Najaf

Luis Neves

Graham Newson

Chayut Ngamkhanong

James Nicholls

Chibuzor Ojum
Roberto Ortega

Grigorios Papageorgiou

Naga Pasupuleti

James Pritchard

Dahai Qi

Yaning Qiao

Amir Rassafi

John Rolt

Maria Rubio Gamez

Umesh Sahoo

Jaroslav Smutny

Richard Spoors

Eric Stannard

Emily Tan

Miles Tight

Rosolino Vaiana

CJ Venter

Caroline Visser

Mohamed Wehbi

Morag White

Iswandaru Widyatmoko

Robin Workman

David Worsley

Shaopeng Wu

Yok Hoe Yap

Semsi Yazici

Yu-Jiang Zhang

Sien Zhou

Fang Zong

Yajie Zou 